\title{
SUBADJOINT IDEALS AND HYPERPLANE SECTIONS
}

\author{
NADIA CHIARLI AND SILVIO GRECO \\ (Communicated by Wolmer V. Vasconcelos) \\ In memory of Mario Raimondo
}

\begin{abstract}
We study the behaviour of the notion of "sub-adjoint ideal to a projective variety" with respect to general hyperplane sections. As an application we show that the two classical definitions of sub-adjoint hypersurface given respectively by Enriques and Zariski are equivalent.
\end{abstract}

\section{INTRODUCTION AND RESULTS}

Let $X \subset \mathbb{P}:=\mathbb{P}^{r}$ be a closed integral sub-variety of the $r$-dimensional projective space over an algebraically closed field $k$ of characteristic zero. Let $J$ be a coherent sheaf of ideals of $\mathcal{O}_{X}$ and let $Z:=V(J)$ be the closed subscheme of $X$ corresponding to $J$. Denote by $V$ (resp. $D$ ) the pull-back of $Z$ (resp. of a general hyperplane section of $X$ ) via the normalization morphism $\nu: \bar{X} \rightarrow X$.

(Here by "general element of a linear system" we mean "all divisors corresponding to the closed points of a suitable non-empty open set of the projective space parametrizing it".)

We recall the following (see [C2], Definition and Theorem, also for a more general setting):

Definition 1. Let $\mathcal{J} \subset \mathcal{O}_{\mathbb{P}}$ be a coherent sheaf of ideals such that $\mathcal{J O}_{X}=J$. We say that $\mathcal{J}$ (or $J$ ) is sub-adjoint to $X$ if the following equivalent conditions are satisfied:

(i) The map

$$
\rho_{s}: H^{0}(\mathbb{P}, \mathcal{J}(s)) \rightarrow H^{0}\left(\bar{X}, \mathcal{J} \mathcal{O}_{\bar{X}}(s D)\right.
$$

is surjective for $s \gg 0$.

(ii) The map

$$
\sigma_{s}: H^{0}(X, J(s)) \rightarrow H^{0}\left(\bar{X}, J \mathcal{O}_{\bar{X}}(s D)\right.
$$

is surjective for $s \gg 0$.

(iii) $J$ is extended (i.e., by definition, $J=\nu_{*} J \mathcal{O}_{\bar{X}}$ )

A remarkable example of sub-adjoint ideal is the conductor, i.e. the largest extended ideal sheaf of $\mathcal{O}_{X}$ (see Condition (iii)).

Received by the editors April 25, 1994 and, in revised form, October 11, 1994

1991 Mathematics Subject Classification. Primary 14C20, 13C13.

Key words and phrases. Sub-adjoint hypersurface, conductor, hyperplane section.

Work partially supported by GNSAGA-CNR and MURST. 
In fact there are the following classical definitions (Gorenstein $[G]$ for curves, and Zariski [Z1] for hypersurfaces):

Definition 2 (Zariski, Gorenstein). Let $X$ be a hypersurface and $\mathfrak{F}$ the conductor of $\mathcal{O}_{X}$. Let $\mathcal{F}$ be the largest ideal sheaf of $\mathcal{O}_{\mathbb{P}}$ such that $\mathfrak{F}=\mathcal{F} \mathcal{O}_{X}$. A hypersurface $F$ of degree $n$ is said to be (GZ)-sub-adjoint to $X$ if it corresponds to a non-zero element of $H^{0}(\mathbb{P}, \mathcal{F}(n))$. (If $r=2$ Gorenstein [G] uses the term adjoint in place of sub-adjoint.)

We remark that in this case condition (i) holds for every $s>0$ (loc. cit.; see also [C2] for a quick cohomological proof). Thus condition (i) (considered for the first time by Arbarello and Ciliberto [AC] in view of non-trivial examples) is a natural generalization of the notion of "sub-adjointness" considered in Definition 2.

In view of Definition 2 the following definition will be useful:

Definition 3. The non-normal subscheme of $X$ is the closed subscheme corresponding to the conductor of $\mathcal{O}_{X}$ and will be denoted by $W$.

Note that the support of $W$ is exactly the set of non-normal points of X. Note also that $F$ is (GZ)-sub-adjoint to $\mathrm{X}$, according to Definition 2 , if and only if $F$ contains $W$ as a subscheme.

Another classical definition of sub-adjoint is due to Enriques [E] in the case of surfaces in $\mathbb{P}^{3}$. We rewrite it from [Z2], p. 53:

Definition 4 (Enriques). Let $F$ be a surface in projective 3-space, with arbitrary singularities. The surfaces $\phi$ which cut out on a general plane adjoint curves of the corresponding plane section $C$ of $F$, are called sub-adjoint surfaces of $F$.

(In modern terms: $\phi$ is sub-adjoint $((E)$-sub-adjoint in the following) if for any general plane $H$ the curve $\phi \cap H$ contains the non-normal subscheme of $C:=X \cap H$.)

Remarks. 1. The notion of "adjoint curve to a plane curve" used in Definition 4 is not defined in Enriques' paper (nor in Zariski's book). Definition 4 is clear and meaningful if one uses Gorenstein's definition, which can be shown to be equivalent to all classical definitions, except the one due to Nöther, which is stronger, and does not seem to be the right one to make things work. (We remark however that for general curves of sufficiently high degree all known definitions of adjoint curve to a given plane curve are equivalent.) For details and bibliography on this matter we refer to [GV1] and [GV2].

2. In the classical literature the notion of sub-adjoint hypersurface to a given hypersurface exists only for surfaces in $\mathbb{P}^{3}$, although it can obviously be given by induction starting from Definition 4. Also the notion of adjoint hypersurface to a given hypersurface exists only for surfaces in $\mathbb{P}^{3}$ (see $[\mathrm{E}]$ or $[\mathrm{Z2}], \mathrm{p}$. 64). There is instead the notion of "adjoint hypersurface to a given space curve" (see [C]), which is a particular case of the situation considered in Definition 1.

The starting point of the present paper is based on the idea of Definition 4, to check sub-adjointness by looking at a general hyperplane section. Indeed one of our motivations is to compare Definitions 2 and 4 , as a starting point toward a modern revisitation of the classical theory of adjoint surfaces of Enriques [E], which is far from being completely understood (the only recent papers on the subject are, to the best of our knowledge, $[\mathrm{BL}]$ and $[\mathrm{S}])$.

Our main result, to be proved in the next section, is the following "going up" and "going down" theorem for sub-adjointness, with respect to hyperplane sections: 
Theorem. Let the notation and the assumptions be as before, with $\operatorname{dim}(X) \geq 2$. Let $X^{\prime}:=H \cap X$ be a general hyperplane section of $X$. Then we have:

(i) $X^{\prime}$ is integral and $\nu^{-1}\left(X^{\prime}\right) \stackrel{\nu}{\rightarrow} X^{\prime}$ is the normalization of $X^{\prime}$;

(ii) If $J$ is sub-adjoint to $X$, then $J^{\prime}:=J_{\mid X^{\prime}}$ is sub-adjoint to $X^{\prime}$;

(iii) If $X$ is $S_{2}$ and $J^{\prime}$ is sub-adjoint to $X^{\prime}$ (for almost all $H$ 's), then $J$ is subadjoint to $X$;

(iv) If $\mathfrak{F}$ is the conductor of $\mathcal{O}_{X}$, then $\mathfrak{F}^{\prime}:=\mathfrak{F} \mathcal{O}_{X^{\prime}}$ is the conductor of $\mathcal{O}_{X^{\prime}}$;

(v) If $X$ is $S_{2}, Z:=V(J)$ has no zero-dimensional components and $J^{\prime}=\mathfrak{F}^{\prime}$ is the conductor of $\mathcal{O}_{X^{\prime}}$ (for almost all $H$ 's), then $J=\mathfrak{F}$ (the conductor of $\mathcal{O}_{X}$ );

(vi) If a hypersurface $F$ is (GZ)-sub-adjoint to $X$, then $F^{\prime}:=F \cap H$ is (GZ)sub-adjoint to $X^{\prime}$;

(vii) If $X$ is $S_{2}$ and $F$ is a hypersurface such that $F^{\prime}$ is (GZ)-sub-adjoint to $X^{\prime}$ (for almost all $H^{\prime}$ s), then $F$ is (GZ)-sub-adjoint to $X$.

Corollary. If $X$ is a surface in $\mathbb{P}^{3}$, then a surface $F$ is (GZ)-sub-adjoint to $X$ if and only if it is (E)-sub-adjoint to $X$, i.e. the definitions of sub-adjoint given by Zariski-Gorenstein and Enriques are equivalent.

\section{ProOFs}

Notation and general assumptions are as in section 1. We begin with the proof of part (i) of the Theorem, which we restate as follows:

Lemma 1. (i) $X^{\prime}$ is an integral scheme;

(ii) $\nu^{-1}\left(X^{\prime}\right)$ is normal;

(iii) $\nu^{-1}\left(X^{\prime}\right) \stackrel{\nu}{\rightarrow} X^{\prime}$ is the normalization of $X^{\prime}$.

Proof. (i) follows from Bertini's Theorem (see e.g. [J], 6.11, 2) and 3), with $d=1$ ).

(ii) follows from $[\mathrm{CGM}]$ (see Theorem 1 and the following Corollary 1, with $\mathcal{P}$ $=$ Normal).

(iii) A general hyperplane $H$ does not contain any irreducible component of the non-normal subscheme of $X$. Hence $\nu$ induces a finite birational map $\nu^{1}\left(X^{\prime}\right) \rightarrow X^{\prime}$ : the conclusion follows from (ii).

In order to prove the rest of the Theorem we need several Lemmas. As usual we denote by an upper bar the integral closure of a domain. If $A$ is a domain, an ideal $I$ of $A$ is said to be extended iff $I \bar{A}=I$ (see Definition 1, (iii)).

Lemma 2. Let $(A, \mathfrak{m})$ be a local noetherian integral domain, with finite integral closure $\bar{A}$ and let $I \subset \mathfrak{m}$ be an ideal of $A$. Let $t \in \mathfrak{m}$ and put $A^{\prime}:=A / t A$ and $I^{\prime}:=I A^{\prime}$. Assume:

a) $A^{\prime}$ is a domain and $\bar{A} / t \bar{A}=\overline{A^{\prime}}$;

b) $t$ is a non zero-divisor $\bmod I$ and $\bmod I \bar{A}$;

Then we have:

(i) $I^{\prime} \overline{A^{\prime}}=I \bar{A} / t(I \bar{A})$;

(ii) $I=I \bar{A} \Longleftrightarrow I^{\prime}=I^{\prime} \overline{A^{\prime}}$ (i.e. $I$ is extended iff $I^{\prime}$ is extended).

Proof. By a) we have

$$
I^{\prime} \overline{A^{\prime}}=I \bar{A}\left(\frac{\bar{A}}{t \bar{A}}\right)=\frac{I \bar{A}}{I \bar{A} \cap t \bar{A}}
$$


and by b)

$$
\frac{I \bar{A}}{I \bar{A} \cap t \bar{A}}=\frac{I \bar{A}}{t(I \bar{A})}
$$

This proves (i).

Now, if $I=I \bar{A}$ we have by (i) and b):

$$
I^{\prime} \overline{A^{\prime}}=I \bar{A} / t(I \bar{A})=I / t I=I / t A \cap I=I^{\prime} .
$$

Conversely if $I^{\prime}=I^{\prime} \overline{A^{\prime}}$, then:

$$
I \bar{A} / t(I \bar{A})=I^{\prime} \overline{A^{\prime}}=I^{\prime}=I / t I
$$

whence $I+t(I \bar{A})=I \bar{A}$, and $I=I \bar{A}$ by Nakayama's Lemma.

Lemma 3. Let the assumptions be as in Lemma 2 and assume further that $A$ is $S_{2}$. If $I^{\prime}$ is the conductor of $A^{\prime}$, then $I$ is the conductor of $A$.

Proof. Let $\mathfrak{b}$ be the conductor of $A$. By Lemma $2 I$ is extended and hence $I \subset \mathfrak{b}$, whence $I^{\prime} \subset \mathfrak{b} A^{\prime}$. Since $A$ is $S_{2}$, all the prime ideals associated to $\mathfrak{b}$ have height 1 , and hence they are associated to $I$. Then by assumption b) $t$ is a non-zero-divisor modulo the conductor (see e.g. [M], Th. 6.5). Then by Lemma 2 applied to $\mathfrak{b}$ we have that $\mathfrak{b} A^{\prime}$ is extended, and hence $\mathfrak{b} A^{\prime} \subset I^{\prime}$, so $\mathfrak{b} A^{\prime}=I^{\prime}$ by the previous remark. Then the inclusion $I \hookrightarrow \mathfrak{b}$ induces an isomorphism $I / t I \rightarrow \mathfrak{b} / t \mathfrak{b}$, whence $\mathfrak{b}=I+t \mathfrak{b}$, and $I=\mathfrak{b}$ by Nakayama's Lemma.

Lemma 4. Let $R$ be a noetherian domain, and let $\mathfrak{a}, \mathfrak{b}$ be two ideals of $R$. Assume that there are maximal ideals $\mathfrak{m}_{1}, \ldots, \mathfrak{m}_{n}$ such that:

a) $\mathfrak{a} R_{\mathfrak{m}_{i}}=\mathfrak{b} R_{\mathfrak{m}_{i}}$ for $i=1, \ldots, n$;

b) every prime ideal associated to either $\mathfrak{a}$ or $\mathfrak{b}$ is contained in some $\mathfrak{m}_{i}$.

Then $\mathfrak{a}=\mathfrak{b}$.

Proof. Let $S=R \backslash \bigcup \mathfrak{m}_{i}$, and let $B:=S^{-1} R$. Then from a) it follows that $\mathfrak{a} B_{\mathfrak{m}_{i} B}=\mathfrak{b} B_{\mathfrak{m}_{i} B}$ for $i=1, \ldots, n$. Then if $M:=(\mathfrak{a} B+\mathfrak{b} B) / \mathfrak{b} B$ we have $M_{\mathfrak{m}}=0$ for every maximal ideal $\mathfrak{m}$ of $B$, whence $M=0$ (e.g. [AM], Prop. 3.8) and $\mathfrak{a} B \subseteq \mathfrak{b} B$. By a similar argument we get the opposite inclusion, whence $\mathfrak{a} B=\mathfrak{b} B$. Moreover by b) no prime ideal associated to either $\mathfrak{a}$ or $\mathfrak{b}$ intersects $S$, and hence (e.g. [AM], Prop. 4.9) $\mathfrak{a}=R \cap \mathfrak{a} B=R \cap \mathfrak{b} B=\mathfrak{b}$.

Lemma 5. Assume $X$ is $S_{2}$ and $\operatorname{dim} X \geq 2$; let $Y \subset X$ be a closed subscheme of codimension at least 2. Then there is a hypersurface $F$ containing $Y$ such that:

a) $F \cap X$ is an integral scheme;

b) $F$ does not contain any irreducible component of the non-normal subscheme $W$ of $X$ (see Def. 2).

Proof. Let $\Sigma_{n}$ be the linear system cut out on $X$ by the hypersurfaces of degree $n$ containing $Y$. If $n$ is sufficiently large, $\Sigma_{n}$ has no base points outside of $Y$ and this easily implies that $\Sigma_{n+1}$ separates points outside of $Y$. Thus $\Sigma_{n+1}$ induces an injective morphism $\phi: X \backslash Y \rightarrow \mathbb{P}^{q}$ (for suitable $q$ ), whence $\operatorname{dim}(\phi(X \backslash Y))=$ $\operatorname{dim}(X) \geq 2$. Hence Bertini's Theorem (e.g. $[\mathrm{J}], 6.11,2)$ and 3), with $d=1$ ) implies that if $E$ is a general element of $\Sigma_{n+1}$, then $E \cap(X \backslash Y)$ is integral. By property $S_{2} E$ is of pure codimension 1 , and since $Y$ has codimension $\geq 2$, it follows that $E$ 
is irreducible. Moreover by Lemma 4 (applied, locally, to the ideals of $E$ and $E_{\text {red }}$ ) we get $E=E_{r e d}$, and then $E$ is an integral subscheme of $X$. Hence for any general hypersurface $F$ of degree $n+1$ containing $Y, F \cap X$ is an integral scheme. Now since all irreducible components of $W$ are 1-codimensional, while $\operatorname{codim}(Y) \geq 2$, it is always possible to choose the hypersurface $F$ so that it does not contain any component of $W$.

Lemma 6. Let $F$ be a hypersurface such that:

a) $F$ does not contain any irreducible component of $Z=V(J)$;

b) $F \cap X$ is integral;

c) $J_{x}$ is extended for all $x \in X \backslash F \cap X$.

Then $J$ is extended.

Proof. The property of being extended is local (e.g. [C2], Theorem), and hence we have to prove the following:

$\left(^{*}\right)$ Let $U:=\operatorname{spec}(R)$ be a standard open affine subscheme of $X$, and $\mathfrak{a}=H^{0}(U, J)$. Then $\mathfrak{a}=\mathfrak{a} \bar{R}$.

We prove first that, if $f \in R$ is an equation of $F \cap U$, then

$$
R_{f} \cap \bar{R}=R .
$$

Indeed let $x \in R_{f} \cap \bar{R}$, and put $x=\frac{a}{f^{n}}$, with $a \in R$ and $n$ minimum. Since $x$ is integral over $\mathrm{R}$, we have an equality of the form

$$
a_{0} f^{n p}+a_{1} f^{n(p-1)}+\ldots+a_{p-1} f^{p}+a^{p}=0
$$

with $p>0$ and $a_{0}, \ldots, a_{p-1} \in R$.

If $n>0$ we have $a^{p} \in(f)$, and since $(f)$ is prime by b), it follows that $a \in(f)$, which contradicts the minimality of $n$. Thus $n=0$, and $x \in R$. Then (**) follows.

Now from a) and c) we get $\mathfrak{a} R_{f} \cap R=\mathfrak{a}$ and $\mathfrak{a} R_{f}=\mathfrak{a} \bar{R}_{f}$. Then, using also (**), it follows that

$$
\mathfrak{a} \bar{R} \subset\left(\mathfrak{a} \bar{R}_{f}\right) \cap \bar{R}=\left(\mathfrak{a} R_{f}\right) \cap \bar{R}=\left(\mathfrak{a} R_{f}\right) \cap R_{f} \cap \bar{R}=\left(\mathfrak{a} R_{f}\right) \cap R=\mathfrak{a},
$$

whence $(*)$, and the conclusion follows.

Now we can proceed in the proof of our Theorem.

Proof of part (ii) of the Theorem. If $H$ is a general hyperplane, then (i) holds, and moreover $H$ does not contain any irreducible component of $Z=V(J)$, and $\nu^{-1}\left(X^{\prime}\right)$ does not contain any irreducible component of $\nu^{-1}(Z)$. Let now $x \in X^{\prime}$, $A:=\mathcal{O}_{X, x}, I:=J_{x}$ and let $t \in A$ be an equation of $H$ near $x$. Then, by Lemma $2, J_{x}^{\prime}$ is an extended ideal of $A^{\prime}=\mathcal{O}_{X^{\prime}, x}$, whence $J^{\prime}$ is extended and the conclusion follows (see Definition 1, (iii)).

Proof of part (iii) of the Theorem. Observe that $J_{x}$ is trivially extended for all $x \in \operatorname{Norm}(X)$. Let now $W_{1}, \ldots, W_{n}$ be the irreducible components of $W$ (the nonnormal subscheme of $X$, see Def. 3). Since $X$ is $S_{2}$, each $W_{i}$ has codimension 1 , and hence dimension $\geq 1$. Then, if $H$ is a general hyperplane, we have $W_{i} \cap H \neq \emptyset$ for all $i$ 's, and hence by Lemma 2 there is $x_{i} \in W_{i}$ such that $J_{x_{i}}$ is extended $(i=1, \ldots, n)$. Then

$$
Y:=\operatorname{Supp}_{X}\left(\nu_{*}\left(J \mathcal{O}_{\bar{X}}\right) / J\right)=\left\{x \in X \mid J_{x} \text { is non-extended }\right\}
$$


is a closed subset of $X$ of codimension at least 2. The conclusion is then a straightforward consequence of Lemmas 5 and 6 (a posteriori $Y$ is empty).

Proof of part (iv) of the Theorem. Since the problem is local, it is sufficient to prove the following:

$\left(^{*}\right)$ let $U=\operatorname{spec}(R)$ be an affine standard open subscheme of $X$, and $\mathfrak{b}$

the conductor of $R$; let $H$ be a general hyperplane, $t \in R$ the equation of $H \cap U$, $R^{\prime}=R / t R$, and $\mathfrak{b}^{\prime}$ the conductor of $R^{\prime}$. Then $\mathfrak{b}^{\prime}=\mathfrak{b} R^{\prime}$.

By Lemma $1 R^{\prime}$ is a domain and $\overline{R^{\prime}}=\bar{R} / t \bar{R}$. Moreover $\mathfrak{b}=\operatorname{Hom}_{R}(\bar{R}, R)$ and $\mathfrak{b}^{\prime}=H_{\mathrm{om}^{\prime}}\left(\overline{R^{\prime}}, R^{\prime}\right)$, whence by standard facts (e.g. [M], p. 140, Lemma 2(ii)) we get $\mathfrak{b}^{\prime}=\operatorname{Hom}_{R}\left(\bar{R}, R^{\prime}\right)$. Now from the exact sequence

$$
0 \longrightarrow R \stackrel{t}{\longrightarrow} R \longrightarrow R^{\prime} \longrightarrow 0
$$

we get the exact sequence:

$$
\begin{aligned}
& 0 \longrightarrow \operatorname{Hom}_{R}(\bar{R}, R) \stackrel{t}{\longrightarrow} \operatorname{Hom}_{R}(\bar{R}, R) \longrightarrow \operatorname{Hom}_{R}\left(\bar{R}, R^{\prime}\right) \\
& \longrightarrow \operatorname{Ext}_{R}^{1}(\bar{R}, R) \stackrel{t}{\longrightarrow} \operatorname{Ext}_{R}^{1}(\bar{R}, R)
\end{aligned}
$$

Since $\bar{R}$ is a finite R-module, it follows that $\operatorname{Ext}_{R}^{1}(\bar{R}, R)$ is such, and hence it has only finitely many associated prime ideals ([M], p. 39, Th. 6.5) and then, being $H$ general, $t$ does not belong to any of them. Then $t$ is $\operatorname{Ext}_{R}^{1}(\bar{R}, R)$-regular ([M], p. 38, Th. 6.1) and this readily implies that $\mathfrak{b}^{\prime}=\mathfrak{b} / t \mathfrak{b}$. But $\mathfrak{b} / t \mathfrak{b}=\mathfrak{b} / t R \cap \mathfrak{b}=\mathfrak{b} R^{\prime}$, since $t$ is a non-zerodivisor modulo $\mathfrak{b}$ (being $H$ general), and the conclusion follows.

Proof of part (v) of the Theorem. Let $W_{1}, \ldots, W_{n}$ be the irreducible components of $\mathrm{W}$ (see Definition 3 ), and let $Z_{1}, \ldots, Z_{n}$ be the irreducible components of Z. We have $\operatorname{dim}\left(W_{i}\right)>0$ for $i=1, \ldots, n$ by property $S_{2}$, and $\operatorname{dim}\left(Z_{j}\right)>0$ for $j=1, \ldots, m$ by assumption. Then any hyperplane intersects all $W_{i}$ 's and all $Z_{j}$ 's, and by an argument as in the proof of part (ii) one can apply Lemma 3 to show that there are $x_{i} \in W_{i}$ and $y_{j} \in Z_{j}$ such that $J_{x_{i}}$ is the conductor of $\mathcal{O}_{X, x_{i}}$ and $J_{y_{j}}$ is the conductor of $\mathcal{O}_{X, y_{j}}(i=1, \ldots, n ; j=1, \ldots, m)$. Since the problem is local, the conclusion is a straightforward consequence of Lemma 4.

Proof of part (vi) of the Theorem. It follows immediately from part (iv).

Proof of part (vii) of the Theorem. The set of the homogeneous polynomials of any given degree corresponding to the hypersurfaces $F$ such that $F^{\prime}$ is (GZ)-subadjoint to $X^{\prime}$ for almost all $H^{\prime}$ 's form the homogeneous parts of a homogeneous ideal $\mathcal{A} \subseteq S:=\bigoplus H^{0}\left(\mathbb{P}, \mathcal{O}_{\mathbb{P}}(n)\right)$. We consider the ideal sheaf $I=\tilde{\mathcal{A}} \mathcal{O}_{X}$, and we claim that $I=\mathfrak{F}$.

Indeed by (vi) we have $\mathfrak{F} \subseteq I$; moreover by the definition of $I$ and of (GZ)-subadjoint (see Definition 2) we get $I^{\prime} \subseteq \mathfrak{F}^{\prime}$ for almost all hyperplanes $H$ 's.

Then $I^{\prime}=\mathfrak{F}^{\prime}$, and in particular $I^{\prime}$ is extended. Then by (iii) $I$ is extended and this implies $I \subseteq \mathfrak{F}$. The conclusion follows.

Remark. The only place where we need the characteristic zero assumption is in part (i) of the Theorem (and in particular when we use a result from [CGM], see proof of Lemma 1). It might be interesting to know whether this assumption can be dropped. 


\section{REFERENCES}

[AC] E. Arbarello, C. Ciliberto, Adjoint Hypersurfaces to Curves in $\mathbb{P}^{r}$ following Petri, in "Commutative Algebra" (S. Greco, G. Valla, eds.), Dekker LNPAM, vol. 84, 1983, pp. 121. MR 84c: 14004

[AM] M.F. Atiyah, I.G. MacDonald, Introduction to Commutative Algebra, Addison-Wesley Publ. Comp., 1969. MR 39:4129

[BL] P. Blass, J. Lipman, Remarks on adjoints and arithmetic genera of algebraic varieties, American J. of Math. 101 (1979), 331-336. MR 80j:14017

[C] G. Castelnuovo, Sui multipli di una serie lineare di gruppi di punti appartenenti ad una curva algebrica, Rend. Circ. Mat. Palermo 7 (1893), 89-110.

[C1] N. Chiarli, A sharp Castelnuovo bound for the normalization of certain projective surfaces, in "Algebraic Geometry and Its Applications" (C. Bajaj, ed.), Springer-Verlag New York, Inc, 1994, pp. 145-151. MR 95c:14009

[C2] Sub-adjoint Ideals to Projective Varieties, Ricerche Mat. (to appear).

[CGM] C. Cumino, S. Greco, M. Manaresi, An Axiomatic Approach to the Second Theorem of Bertini, J. of Algebra 98 (1986), 171-182. MR 87k:14008

[E] F. Enriques:, Introduzione alla geometria sopra le superficie algebriche, Mem. Soc. Ital. Sci. detta dei 40, III serie 10 (1896), 1-81.

[G] D. Gorenstein, An Arithmetic Theory of Plane Adjoint Curves, Trans. AMS 72 (1952), 414-436. MR 14:198h

[GV1] S. Greco, P. Valabrega, On the Theory of Adjoints, in "Algebraic Geometry" (K. Lønsted, ed.), Springer-Verlag LNM, vol. 732, 1979, pp. 98-123. MR 82e:14015

[GV2] , On the Theory of Adjoints II, Rend. Circ. Mat. Palermo 31 (1982), 5-15. MR 84a:14005

[J] J.P. Jouanolou, Théorèmes de Bertini et Applications, Progr. in Math., vol. 42, Birkhäuser, 1983. MR 86b:13007

[M] H. Matsumura, Commutative Ring Theory, vol. 8, Cambridge University Press, 1990. MR 88h:13001

[S] E. Stagnaro, Canonical and pluricanonical adjoints I, II, III (Preprints 1991,1992) (1991, 1992).

[Z1] O. Zariski, An Introduction to the Theory of Algebraic Surfaces, Springer LNM, vol. 83, Springer-Verlag, 1969. MR 41:8418

[Z2] Algebraic Surfaces, Springer-Verlag, 1971. MR 57:9695

Dipartimento di Matematica, Politecnico di Torino, Corso Duca degli Abruzzi, 24 10129 TORINO, ITALY

E-mail address: CHIARLI@POLITO.IT

E-mail address: SGRECO@POLITO.IT 